PAPER

\title{
Anosmia is very common in the Lewy body variant of Alzheimer's disease
}

\author{
J M Olichney, C Murphy, C R Hofstetter, K Foster, L A Hansen, L J Thal, R Katzman
}

J Neurol Neurosurg Psychiatry 2005;76:1342-1347. doi: 10.1136/jnnp.2003.032003

See end of article for authors' affiliations

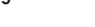

Correspondence to: Dr John M Olichney, Neurology Service (9127),

VA San Diego Healthcare System, 3350 La Jolla Village Drive, San Diego, CA 92161, USA:

olichney@cogsci.ucsd.edu

Received 26 January 2004

In revised form

14 January 2005

Accepted 16 January 2005
Background: Olfactory abnormalities are reported in Alzheimer's disease and Parkinson's disease. Anosmia appears to be common in dementia with Lewy bodies but not in pure Alzheimer's disease. Objective: To determine whether anosmia improves discrimination between the Lewy body variant (LBV) of Alzheimer's disease and "pure" Alzheimer's disease.

Methods: 106 cases of necropsy confirmed pure Alzheimer's disease $(n=89)$ or LBV $(n=17)$ were reviewed. All had received butanol odour threshold testing. Anosmia was defined as a score $\leqslant 1.0$ on a 0-9 point scale. Logistic regression analysis was used to model potential predictors (for example, parkinsonism, smoking, hallucinations) of neuropathological diagnosis and anosmia.

Results: LBV cases had an increased prevalence of anosmia (65\%) compared with Alzheimer's disease $(23 \%$; odds ratio $(O R)=6.3, p=0.00045)$, or normal elderly people $(6.7 \%)$. Within the dementia cases, the negative predictive value $(92 \%)$ and specificity $(78 \%)$ of anosmia were both good; sensitivity for detecting LBV was $65 \%$, but the positive predictive value (PPV) was only $35 \%$. Logistic regression models showed anosmia $(O R=5.4, p=0.005)$ and visual hallucinations $(O R=7.3, p=0.007)$ were strong independent predictors of Lewy body pathology. When anosmia was added as a core feature to consensus diagnostic criteria for probable Lewy body dementia, five additional cases of LBV were detected ( $29 \%$ increased sensitivity), but with four additional false positives ( $1 \%$ increased discrimination, $4 \%$ decreased specificity, 33\% decreased PPV).

Conclusions: Anosmia is very common in LBV. Adding anosmia as a core feature improved sensitivity for detecting LBV, but did not improve discrimination between Alzheimer's disease and LBV owing to a concomitant increase in false positives.
A bnormal olfaction has been well described in clinical cohorts of both Parkinson disease and Alzheimer's disease. ${ }^{1-3}$ McShane and colleagues ${ }^{4}$ first reported that anosmia is increased in dementia cases with Lewy bodies confirmed at necropsy, but not increased among those with pure Alzheimer's disease pathology. It is not surprising that cases with Lewy body dementia would have a high prevalence of anosmia, as these cases greatly resemble Parkinson's disease neuropathologically and are usually phenotypically similar to cases of Parkinson's disease with dementia.

The reliable diagnosis of dementia with Lewy bodies (DLB) remains very difficult during life. The most challenging cases are those with significant amounts of superimposed Alzheimer's disease pathology (which we designate as the "Lewy body variant of Alzheimer's disease", or LBV). Recent work has shown an inverse relation between Braak stage and diagnostic accuracy in LBV. ${ }^{5}$ The current consensus diagnostic criteria ${ }^{7}$ for probable DLB requires that two or more of the following three core features are present: spontaneous motor features of parkinsonism; fluctuating cognition with pronounced variations in attention and alertness; and recurrent visual hallucinations. If only one core feature is present, a diagnosis of "possible DLB" can be made. While McKeith and colleagues ${ }^{8}$ have reported high sensitivity (83\%) and specificity (95\%) when they apply these criteria for probable DLB to their dementia cohort, many Alzheimer's disease research centres have found only mediocre diagnostic accuracy. For example, Verghese et al ${ }^{9}$ found that these criteria had good sensitivity and specificity ( $78 \%$ and $85 \%$ ) in patients with "pure" DLB, but only $44 \%$ sensitivity for cases with DLB and Alzheimer pathology. Similarly, Lopez et al ${ }^{10}$ reported only fair sensitivity $(62 \%)$ and specificity $(54 \%)$ in mild dementia cases. While sensitivity was excellent (93\%) for severe DLB cases, specificity was only $16 \%$ among the other (non-DLB) severe dementia cases. Merdes and colleagues $^{7}$ reported that, among necropsy confirmed DLB cases from our centre, clinical diagnostic accuracy was quite good $(75 \%)$ for cases with DLB with little or no Alzheimer pathology (Braak stages 0-II), but that accuracy fell to 39\% for LBV cases (Braak stages of III or more).

The main objectives of the present study were first, to attempt to confirm the findings of McShane et $a l^{4}$ in our necropsy confirmed cases with LBV; and second, to determine whether adding the sign of anosmia as a core feature to the consensus diagnostic criteria for $\mathrm{DLB}^{7}$ increases their sensitivity or improves diagnostic accuracy in discriminating between Alzheimer's disease and LBV.

\section{METHODS}

\section{Patients}

The study included consecutive dementia cases coming to necropsy between 1988 and 2001 with a primary neuropathological diagnosis of either LBV or Alzheimer's disease. All had undergone semiquantitative odour threshold testing at the Alzheimer's Disease Research Center (ADRC) of the University of California, San Diego (UCSD). Odour threshold

Abbreviations: ADRC, Alzheimer's Disease Research Center; CERAD, Consortium to Establish a Registry for Alzheimer's Disease; DLB, dementia with Lewy bodies; DRS, dementia rating scale; DSM-III R, Diagnostic and Statistical Manual of Mental Disorders, 3rd edition revised; LBV, Lewy body variant; NINCDS-ADRDA, National Institute of Neurological and Communicative Disorders and Stroke and the Alzheimer's Disease and Related Disorders Association; UCSD, University of California, San Diego; UPDRS, unified Parkinson disease rating scale 
testing was generally carried out during either the mild or the moderate stage of the dementia. All selected cases met the CERAD neuropathological criteria ${ }^{11}$ for definite or probable Alzheimer's disease, and the DSM-III R criteria for dementia. ${ }^{12}$ In addition, the LBV group had Lewy bodies present in the brain stem and cerebral cortex. Nearly all these cases satisfied NINCDS-ADRDA clinical criteria for either probable or possible Alzheimer's disease ${ }^{13}$ at the time of olfactory testing. Cases with a clinical diagnosis of Parkinson's disease with motor symptoms for more than 12 months before dementia onset were excluded, as recommended by the consensus clinical criteria for DLB. $^{7}$ While cases with dementia and parkinsonism at entry into the ADRC were not excluded, all the LBV cases had a history of cognitive symptoms preceding any motor symptoms of parkinsonism. To reduce the neuropathological heterogeneity of our LBV group, we excluded cases $(n=3)$ with "pure" DLB (that is, cases without sufficient Alzheimer pathology to meet CERAD neuropathological criteria for definite or probable Alzheimer's disease). ${ }^{14}$ These criteria resulted in a series of 106 cases, 89 with a primary neuropathological diagnosis of Alzheimer's disease and 17 with LBV. All these cases were ADRC participants who had given informed consent for olfactory testing, longitudinal clinical assessments, and brain necropsy, as per protocols approved by the UCSD Human Research Protection Program.

All patients underwent a structured interview and standardised neurological examination by a neurologist which included ascertainment of the following extrapyramidal signs: generalised bradykinesia, resting tremor, rigidity, masked facies, parkinsonian gait, and postural instability. For examinations before 1993, these signs were rated as either absent or present (with a rating scale of +1 for mild to moderate abnormalities and +2 for moderate to severe abnormalities). From 1993 forward (37 cases), these extrapyramidal signs were classified as "present" when the corresponding unified Parkinson disease rating scale (UPDRS) $)^{15}$ score was 2 or more at the examination closest to olfactory testing. Most ADRC subjects have these structured interviews and neurological examinations repeated annually, from enrolment until the year of death. These annual assessments include an extensive neuropsychological test battery which assesses attention, memory, language, visuospatial construction, and problem solving abilities. ${ }^{16}$ Global dementia severity was assessed by the dementia rating scale. ${ }^{17}$ The structured interview included queries of an informant (usually the spouse) regarding fluctuations in cognition and the presence of hallucinations

Table 1 Demographic, clinical, and olfactory data for the patient groups

\begin{tabular}{llll}
\hline & LBV & AD & p Value \\
\hline $\mathrm{n}$ & 17 & 89 & - \\
Sex & $11 \mathrm{M} ; 6 \mathrm{~F}$ & $51 \mathrm{M} ; 38 \mathrm{~F}$ & 0.57 \\
& $64.7 \% \mathrm{M}$ & $57.3 \% \mathrm{M}$ & \\
Age (years) & $74.3(5.7)$ & $73.2(8.3)$ & 0.60 \\
Education (years) & $14.9(2.6)$ & $13.9(3.5)$ & 0.30 \\
DRS & $100.6(20.4)$ & $106.2(21.8)$ & 0.33 \\
No of EPS & $0.71(1.16)$ & $0.29(0.81)$ & 0.07 \\
Current smoker & $11.8 \%$ & $11.9 \%$ & 0.98 \\
History of smoking & $47.1 \%$ & $68.7 \%$ & 0.10 \\
Anosmia & $64.7 \%(11 / 17)$ & $22.5 \%(20 / 89)$ & $0.0004^{*}$ \\
Odour threshold & $1.79(2.42)$ & $4.10(2.62)$ & $0.001^{*}$ \\
Time interval (years), & & & \\
from testing to death & $4.9(3.3)$ & $5.3(2.8)$ & 0.62 \\
\hline
\end{tabular}

Values are mean (SD) or prevalence $(\%)$

*Two sided $\mathrm{p}<0.05$.

$A D$, Alzheimer's disease; DRS, dementia rating scale ${ }^{17}$; EPS,

extrapyramidal signs; $F$, female; LBV, Lewy body variant; $M$, male. (visual or auditory) within the previous year. The presence of visual or other hallucinations was also assessed by a structured interview with an informant, using the diagnostic interview schedule. ${ }^{18}$ Smoking history, which was obtained from a questionnaire given to the informant, included estimates of duration and quantity of cigarette, cigar, and pipe smoking. These data were classified on a four point scale, with the following categories: current smoker; quit within six months; quit over six months ago; never smoked. This form of data reduction was used because smoking has been shown to increase the risk of anosmia, but olfactory function may slowly return to normal after cessation. ${ }^{19}$ Consensus criteria for possible and probable DLB were applied retrospectively by a single investigator (JO), which involved a review of all clinical data acquired up to the time of odour threshold testing, blinded to neuropathological diagnosis. Other details regarding the clinical assessment and diagnostic procedures of the UCSD ADRC have been published previously. ${ }^{20}$

\section{Olfactory stimuli and test procedures}

Olfactory testing was generally done within one week of the annual neuropsychological tests described above. The odorant n-butyl alcohol was prepared in a series of 10 dilutions beginning with a $4 \%$ solution (by volume) in deionised water. $\mathrm{N}$-butyl alcohol is often used in olfactory threshold testing because it is a potent stimulus for the olfactory nerve at concentrations which have no impact on the trigeminal nerve. Each successive dilution was one third the concentration of the preceding dilution. The odour threshold for butanol was determined separately for each nostril, using a two alternative, forced choice task with ascending concentrations, ${ }^{21}$ modified as in Murphy et al..$^{22}$ Each pair of stimuli consisted of a blank and an odour stimulus. The subject sniffed each stimulus and then chose which of the two smelled stronger. In order to minimise the effects of adaptation, testing progressed from weaker to stronger concentrations with approximately 90 seconds between trials. An incorrect choice led to an increased concentration on the next trial. Correct choices led to presentation of the same concentration, to a criterion of four correct choices in a row. Two threshold determinations were made for each subject, one for each nostril, using a 0 to 9 point scale. An average score of $\leqslant 1.0$ was considered "anosmia." This threshold was chosen because $4 \%$ butyl alcohol is often strong enough to be detected by the trigeminal pathway, but this is rarely the case for a $1.33 \%$ solution. The mean odour threshold for 150 normal elderly controls (mean age, 71.6 years) tested at our ADRC was 5.75 ( $\mathrm{SE}=1.7)$. The prevalence of anosmia was $6.67 \%$ in these normal elderly controls, using the same criteria.

\section{Neuropathology}

The full neuropathological procedures of our ADRC and CERAD have been published previously. ${ }^{14} 2324$ All neuropathological measures were made blind to clinical diagnoses and to olfactory and cognitive test scores. Neuritic plaques were assessed on thioflavin-S or Bielchowsky silver stains of cerebral neocortex. All cases in this report had sufficient neuritic plaque density to meet CERAD criteria ${ }^{14}$ for probable or definite Alzheimer's disease. Lewy bodies were detected by either antiubiquitin or haematoxylin and eosin (H\&E) staining. In addition to satisfying CERAD criteria for probable or definite Alzheimer's disease, the presence of one or more Lewy bodies in both the brain stem and cerebral cortex was required for the neuropathological diagnosis of LBV. In none of the LBV cases were Lewy bodies confined to only the amygdala or visible only with $\alpha$-synuclein labelling. Modified Braak staging was carried out on all cases, using procedures we have described previously. ${ }^{24}$ 


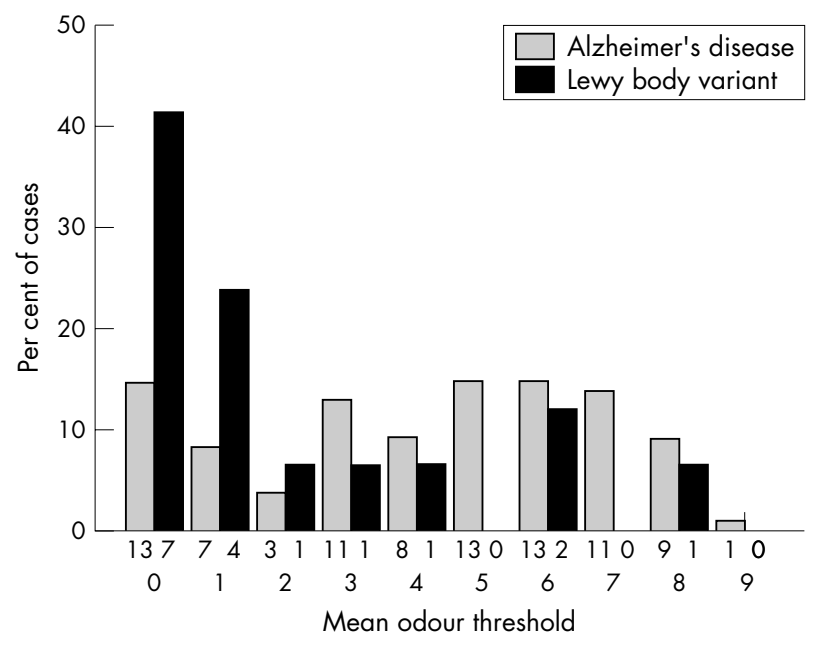

Figure 1 Frequency distribution of mean odour threshold scores (average of right and left nostrils) for the Alzheimer $(n=89)$ and Lewy body variant $(n=17)$ patient groups. The raw number of cases is indicated beneath each bar.

\section{Statistical analyses}

All statistical analyses were done using SPSS (version 6.1.3; SPSS Inc, Chicago, Illinois, USA). Intergroup differences were tested for by either $t$ tests (for continuous variables) or the $\chi^{2}$ statistic (for categorical variables). Pearson's correlation coefficients were used where appropriate to test for bivariate correlations. Two tailed $p$ values of 0.05 or less were considered statistically significant. Logistic regression analyses were carried out, which modelled either the presence of Lewy bodies or anosmia as the dependent variable. The regressions for the presence of Lewy bodies included the independent variables of dementia severity (dementia rating scale (DRS) score), anosmia, age, education, sex, visual hallucinations, cognitive fluctuations, and the number of parkinsonian signs. The regression model for anosmia included the independent variables of neuropathological Lewy bodies, age, dementia severity, education, sex, and visual hallucinations. These models were also conducted including the variables of time interval (from olfactory testing to death), smoking status, and Braak stage. Generally, backward step regressions were used (probability-out $=0.10$ ). However, forward step multiple linear regression analyses were conducted (probability-in $=0.10$ ) to model odour threshold score using the presence of Lewy bodies, Braak stage (neurofibrillary numerical staging score and amyloid burden letter score as two separate variables), age, sex, education, DRS total score, and time interval as independent variables. This allowed us to test whether a poor olfactory threshold was most strongly associated with a shorter survival interval, more severe cognitive impairment, or greater Alzheimer's disease pathological burden (either neurofibrillary or amyloid).

\section{RESULTS}

The demographic and clinical characteristics of the patient groups are shown in table 1. The LBV and Alzheimer groups were very similar with respect to age, sex, and educational level. There was no significant group difference in severity of dementia, as measured by the total DRS score near the time of olfactory threshold testing (mean DRS $=100.6$ in LBV and 106.2 in Alzheimer's disease; $t$ test, $\mathrm{p}=0.33$ ). While there was a larger mean number of extrapyramidal signs in LBV than in Alzheimer's disease, the number of extrapyramidal signs present was modest in LBV (mean (SD), 0.71 (1.16)) and the intergroup comparison failed to reach statistical significance. For comparison, the three cases of "pure" DLB had a mean of 1.67 extrapyramidal signs at the time of olfactory testing. There was no significant difference in the smoking history of the two groups, although there was a trend toward more ex-smokers in the Alzheimer group (table 1).

Anosmia was very common in LBV, present in $64.7 \%$ of LBV cases compared with $22.5 \%$ in Alzheimer's disease-a highly significant group difference $\left(\chi^{2}=12.3 ; p=0.00045\right)$. If we had included the three cases with "pure" DLB at necropsy in this report, the results would be nearly identical (two of three had anosmia, meaning that $65 \%$ of the total LBV/DLB sample had anosmia). The mean (SD) odour threshold score was 4.0 (2.6) in Alzheimer's disease, but only 1.8 (2.4) in LBV ( $t$ test, $\mathrm{p}=0.001$ ). The distributions for olfactory threshold in LBV and Alzheimer's disease are shown in fig 1 (all scores are rounded to the nearest integer; therefore scores of 1.5 were combined with 2.0 for the histogram figure). There was a modest correlation between odour threshold and DRS score $(r=0.20, \mathrm{p}=0.04)$, which was somewhat stronger $(r=0.48)$ and of marginal significance $(p=0.052)$ within the LBV cases. Only $23 \%$ of cases with anosmia (7/31) had one or more extrapyramidal signs, which was not significantly different than the prevalence of extrapyramidal signs in cases without anosmia (16\%; $\chi^{2}=0.65, \mathrm{p}=0.42$ ). The relatively low prevalence of extrapyramidal signs is not entirely unexpected, because patients with a clinical diagnosis of Parkinson's disease were excluded. Eighteen per cent of the entire cohort had at least one extrapyramidal sign. There also was no significant relation between anosmia and the number of extrapyramidal signs present $\left(\chi^{2}=1.76, \mathrm{p}=0.78\right)$.

The odour threshold test, by itself, achieved $65 \%$ sensitivity and $78 \%$ specificity for LBV in our cohort (table 2, first row). While the negative predictive value (NPV) was high (92\%), the positive predictive value (PPV) was only $35 \%$ (the pre-test probability of LBV was $16 \%$ in this sample). There was no significant relation between anosmia and the Alzheimer pathological stage in LBV. The proportion of cases with anosmia was $60 \%$ (3/5) for Braak ${ }^{25}$ stages I-II, $60 \%$ for stages III-IV, and 71\% (5/7) for stages V-VI. When we required that only anosmia in non-smokers be considered a "pathological" risk factor for LBV, the sensitivity fell from $65 \%$ to $29 \%$ overall (5/17), but with improvements in specificity (92\%), PPV, and overall discrimination (table 2; compare rows 1 and 2).

The consensus criteria for probable DLB (which requires the presence of two or more core features) had $100 \%$ specificity and PPV (3/3) in our cohort, but the NPV was less impressive (89/103), with low sensitivity (3/17). It should be kept in mind that this very low sensitivity partly reflects, first, that these criteria were applied when the patients were mostly in the mild stage of dementia; second, that our ADRC largely comprises referred patients with an Alzheimer's disease-like phenotype; and third, that any cases diagnosed with Parkinson's disease before dementia onset were excluded. Sensitivity would have been higher if we had taken the last clinical diagnosis before death, as is customary in cliniconeuropathological studies. Next, we tested the extent to which adding anosmia as a core feature might improve the diagnostic accuracy of established consensus criteria $^{8}$ for the diagnosis of DLB. Adding anosmia resulted in the detection of five additional probable DLB cases, but at the cost of four additional false positive cases. While overall diagnostic accuracy was not significantly improved, sensitivity increased by $29 \%$, with a fall in specificity of $4 \%$ (table 2 , compare rows 4 and 5). The less stringent criteria for possible DLB resulted in somewhat improved sensitivity (9/17), but 
Table 2 Diagnostic accuracy of anosmia and other clinical criteria for the detection and discrimination of Lewy body variant cases

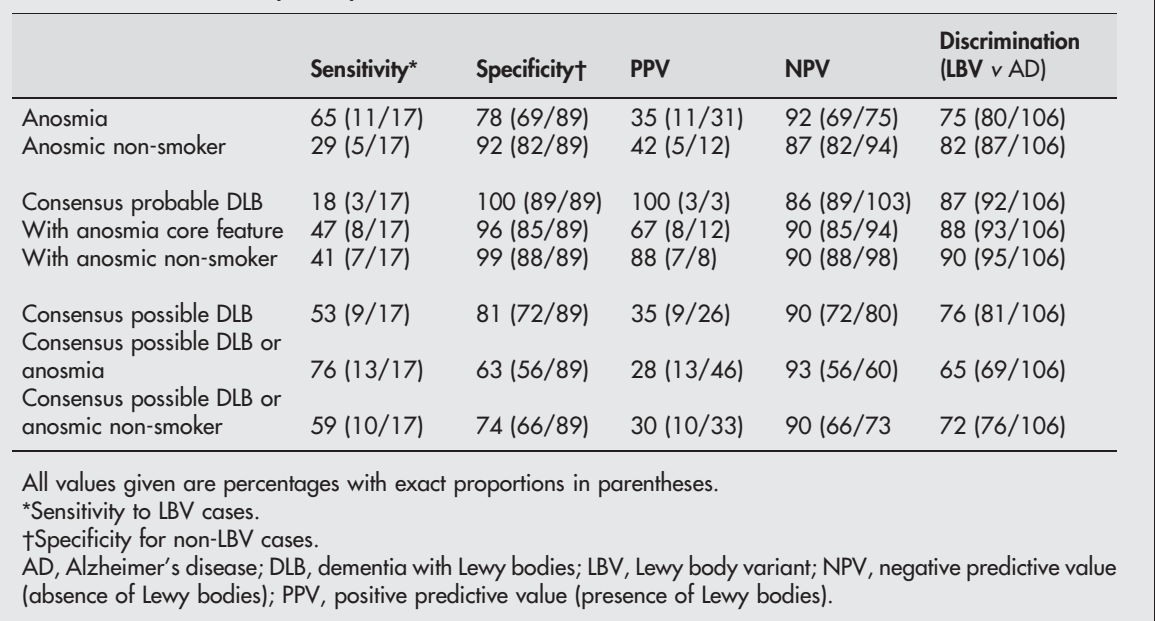

lower overall discrimination $(76 \%)$, specificity $(81 \%)$, and PPV (35\%). Adding anosmia as a core feature, sufficient for the diagnosis of possible DLB \% (table 2, row 7), resulted in good sensitivity $(76 \%)$ but mediocre specificity $(63 \%)$, a low PPV $(28 \%)$, and an overall diagnostic accuracy of only $65 \%$. The highest diagnostic accuracy overall (90\%) was achieved by adding anosmia in a non-smoker as a core feature to consensus criteria for probable DLB \% (row 5 of table 2), which produced an excellent specificity (88/89), high PPV (7/ $8)$, but only mediocre sensitivity (7/17).

The logistic regressions modelling the neuropathological presence of Lewy bodies showed that only anosmia (odds ratio $(\mathrm{OR})=5.4$ (95\% confidence interval $(\mathrm{CI}), 1.67$ to 30.12 ), $\mathrm{p}=0.0049)$ and visual hallucinations $(\mathrm{OR}=7.3$ (1.71 to 31.08), $p=0.0072$ ) were strong independent predictors for LBV. The number of parkinsonian signs, keeping in mind that cases with a clinical diagnosis of Parkinson's disease were excluded, was not a significant independent predictor.

The regression models for anosmia showed three significant independent variables: neuropathological Lewy bodies $(\mathrm{OR}=5.67(95 \% \mathrm{CI}, 1.75$ to 18.09$), \mathrm{p}=0.0035)$, low DRS score $(\mathrm{OR}=0.98(0.96-1.00), \mathrm{p}=0.049)$, and high education $(\mathrm{OR}=1.24(1.06-1.45), \mathrm{p}=0.0066)$. This relation between anosmia and higher education could reflect the fact that such individuals have a greater neuropathological burden by the time they become demented. ${ }^{26}$ When the time interval from olfactory testing to death was added as an independent variable, it remained in the model $(B=-0.24$ (95\% CI, -0.42 to -0.05$), \mathrm{p}=0.01$ ) replacing DRS score. This is likely to reflect the co-linear relation present between DRS and subsequent survival $(r=0.33, \mathrm{p}=0.001)$.

The multiple linear regression models for odour threshold score showed that a shorter time interval before death ( $\mathrm{B}=0.31$ (95\% CI, 0.15 to 0.48$), \mathrm{p}=0.0003)$, the presence of Lewy bodies $(B=-2.07(-3.35$ to -0.79$), p=0.002)$, and high education $(\mathrm{B}=-0.12(-0.026$ to 0.015$), \mathrm{p}=0.08)$ were all significant predictors of poorer (lower) odour threshold scores for the entire patient group.

Comparisons of the pure Alzheimer's disease cases with anosmia versus those without by $t$ test showed no intergoup difference in Braak stage (for example, mean neurofibrillary pathology stage $=5.50 \vee 5.48 ; \mathrm{p}=0.95)$. Pure Alzheimer patients with anosmia tended to survive a shorter period than Alzheimer patients without anosmia (means: $4.02 \vee 5.65$ years; $\mathrm{p}=0.019$ ). Analogous $t$ tests in LBV showed no significant differences in Braak stage (means: 3.82 v 3.17; $\mathrm{p}=0.49$ ) or survival interval (means: $4.4 v 5.9$ years; $\mathrm{p}=0.37)$ between those with anosmia $(\mathrm{n}=10)$ and those without anosmia $(n=7)$. A trend for lower DRS scores in the anosmic LBV cases $(94.3(22.6) v 112.3$ (7.3) in those without anosmia) did not reach statistical significance $(t=1.88$, $\mathrm{p}=0.08$ ), perhaps owing to the small sample size.

\section{DISCUSSION}

Anosmia was a very common finding, present in nearly two thirds of our necropsy confirmed LBV cases. This is the second clinicopathological study we are aware of that has reported increased anosmia in dementia cases with Lewy bodies, and the first to quantify threshold with a rigorous psychophysical procedure that corrects for response bias. McShane $e t ~ a l^{4}$ reported that nine of their 22 dementia cases with Lewy bodies $(41 \%)$ had anosmia, using a $1.7 \%$ lavender oil solution as their stimulus. Sixteen $(73 \%)$ of their Lewy body cases also met CERAD criteria for probable or definite Alzheimer's disease, and they found a similar prevalence of anosmia in pure DLB and in Lewy body cases with superimposed Alzheimer pathology (which we label as "Lewy body variant"). Using a butanol odour threshold test, we found $65 \%$ of LBV cases had anosmia. This suggests that anosmia is one of the most common clinical signs in LBV, a patient group which remains difficult to diagnose when only minimal extrapyramidal signs are present early in the disease course.$^{10}$ In this regard, it should be noted, however, that anosmia appeared to be relatively independent of extrapyramidal signs in our dementia cohort which excluded cases with a clinical diagnosis of Parkinson's disease (for example, there was no relation between anosmia and the number of extrapyramidal signs present). A limitation of the present study is that most patients did not receive the UPDRS at the time of olfactory testing. Thus patient examinations before 1993 may not have been as sensitive to mild extrapyramidal signs as are cut off scores of $\geqslant 2$ on motor UPDRS items. Furthermore, we did not consider UPDRS scores of 1 as definite extrapyramidal signs as they are common in both Alzheimer's disease and elderly normal control groups. Previous studies in Parkinson's disease have also found that olfactory deficits appear to be independent of disease duration and the severity of extrapyramidal signs. ${ }^{27}$ Likewise, we found anosmia and visual hallucinations to be independent predictors of Lewy bodies. Thus adding anosmia to the established consensus diagnostic criteria is likely to increase sensitivity markedly, even in cohorts with relatively mild dementia. 
Despite the high prevalence of anosmia in LBV, we did not find that this symptom in itself produced satisfactory discrimination between LBV and Alzheimer's disease. When we added anosmia as a core feature to the consensus diagnostic criteria for probable DLB, we found-as did McShane et $a l^{4}$-an increase in sensitivity with a decreased specificity and little change in overall diagnostic accuracy. We were able to achieve the highest diagnostic accuracy when we only considered the finding of anosmia in those without a history of smoking as a core feature for the criteria for probable DLB. While these modified criteria had around $90 \%$ accuracy in discriminating between LBV and pure Alzheimer's disease, and a good PPV (7/8), the sensitivity was only modest (7/17). Also, the overall accuracy was not significantly better than that of the established criteria for probable DLB $(87 \%)$. These criteria are conservative and resulted in most of the LBV cases being classified as Alzheimer's disease, but did achieve a PPV of $100 \%$. Perhaps discrimination could be improved further by specialist evaluations to rule out other common medical aetiologies of anosmia in the elderly (for example, nasal disease, paranasal sinus disease, viral infection, trauma). ${ }^{28}{ }^{29}$

An argument could well be made that unexplained anosmia might be more appropriate as a supportive feature than a core feature for DLB. It should be noted that for several purposes (for example, enrolling large samples of LBV patients for clinical drug trials; or if specific highly effective treatments are found for LBV), it could be advantageous to improve detection sensitivity, even at substantial loss of specificity. In this regard, we achieved the highest sensitivity (76\%) when we added anosmia to the criteria for possible DLB, but at the cost of a low positive predictive value.

Caution is advised in applying these findings to other cohorts, such as those with Parkinson's disease. Reliable data on sensitivity, specificity, and the positive and negative predictive value of a diagnostic tool can only be obtained in a cohort that is representative of the population in which it is to be used. Our ADRC is a referral centre for dementia cases, most of whom have an Alzheimer's disease-like phenotype. If anosmia were used to discriminate LBV from our normal elderly, for example, 65\% sensitivity and 93\% specificity would be attained.

Regarding the underlying pathophysiology of anosmia in LBV, it seems likely that the Lewy body burden in areas such as the anterior olfactory nucleus, orbitofrontal, and anterior cingulate cortices could account for the increased anosmia in LBV. Neuropathological changes in the amygdala and entorhinal cortex are particularly severe in LBV, both of these regions often having spongiform neuropil vacuolisation. ${ }^{30}$ Braak et al showed that the anterior olfactory nucleus is one of the main predilection sites for $\alpha$-synuclein pathology in the earliest stages of Parkinson's disease. ${ }^{31}$ By the middle stages, Lewy body pathology begins to appear as well in the piriform, entorhinal, and other olfactory cortices. Anosmia in LBV is unlikely to be primarily a result of Alzheimer's disease pathology. Specifically, neurofibrillary tangles are much less abundant and less widespread in LBV than in pure Alzheimer's disease. ${ }^{32}$ As in the results of McShane et al, ${ }^{4}$ we found no relation between the Braak stage of Alzheimer pathology and anosmia in our LBV sample. However, McShane and colleagues did find that anosmia was related to higher cortical Lewy body scores and to greater Lewy body density in the cingulate gyrus.

While anosmia was not as common in Alzheimer's disease as in LBV, hyposmia was quite common in Alzheimer's disease (mean olfactory threshold $4.10 \vee 5.75$ in elderly controls). Several other studies have shown impaired odour threshold in Alzheimer's disease using standard psychophysical measurements. ${ }^{22}{ }^{23}$ Our analyses suggest that it was the fairly common finding of anosmia in Alzheimer's disease that limited our ability to discriminate LBV from Alzheimer cases more accurately. Discrimination was improved somewhat by only considering anosmia in non-smokers to be a likely sign of a neurodegenerative disorder such as LBV or DLB. The $22.5 \%$ prevalence of anosmia in our Alzheimer cohort is in line with the $16 \%$ prevalence reported by McShane et al. ${ }^{4}$ It has been shown in a cohort with "questionable Alzheimer's disease" (comparable to mild cognitive impairment ${ }^{34}$ ) that smell identification tests are more sensitive to early Alzheimer pathology than are odour threshold tests. ${ }^{35}$ Not only is odour identification impaired in mild Alzheimer's disease, ${ }^{35}{ }^{36}$ but it has been recently shown to be reduced in carriers of the apolipoprotein $€ 4$ allele, who have an increased genetic risk of Alzheimer's disease. ${ }^{37}$ Those at genetic risk show a decline in odour identification over time ${ }^{38}$ and those at risk for Alzheimer's disease (because of mild cognitive impairment) and who have both impaired odour identification and an unawareness of their deficits are more apt to go on to develop the disease. ${ }^{39}$ Patients with $\mathrm{LBV}^{40}$ and diffuse Lewy body disease ${ }^{41}$ have also been reported to show impairments in odour identification. Odour identification was introduced at our ADRC subsequent to odour threshold and odour memory testing and thus is not available for many of the cases reported here. The lack of odour identification testing in all of our LBV cases is a limitation of the present study.

Some previous studies in Alzheimer cohorts have found that anosmic patients have somewhat greater disease severity than patients without anosmia. ${ }^{22}$ In the present study we also found a relation between anosmia and dementia severity, but the correlation was modest in the entire cohort, and driven mostly by the LBV group. Other previous studies of olfactory threshold in clinical Alzheimer cohorts, without neuropathological confirmation, have reported that both anosmia ${ }^{33}$ and a fast decline in olfactory sensitivity ${ }^{42}$ were associated with more rapid progression of dementia. It is unknown whether some of these patients may have had LBV or if anosmia in Alzheimer's disease reflects more severe Alzheimer pathology in the olfactory cortices. Our present results favour the latter, in that Alzheimer cases with anosmia had shorter survival than cases without anosmia, and poor odour thresholds were associated with shorter survival in the entire (predominantly Alzheimer's disease) cohort. Fully resolving this issue will require larger quantitative clinicopathological studies. Longitudinal characterisation of olfactory deficits (for example, anosmia, hyposmia, or olfactory naming deficits) may provide useful clinical measures for tracking disease progression in patients with mild Alzheimer's disease with high education and premorbid intellectual abilities, factors that decrease the sensitivity of conventional neuropsychological tests.

\section{Conclusions}

The present study, although limited in sample size, suggests that anosmia may be one of the most common clinical findings in cases with necropsy confirmed LBV. While anosmia is fairly sensitive to LBV, it is also occasionally present in mild Alzheimer's disease, which limits its predictive value in discriminating between these two disorders. The present study is limited by sample size, and further research of olfactory function in larger dementia cohorts with necropsy data appears warranted in an attempt to improve the detection of DLB. Diagnosing DLB during life is particularly challenging when there is significant concomitant Alzheimer's disease pathology. ${ }^{6}$ Anosmia deserves further consideration as a supportive feature or core feature to aid in the diagnosis of LBV or DLB. 


\section{ACKNOWLEDGEMENTS}

Supported by grants from the National Institutes of Health (AG04085, AG08203, P50 AG05131, and AG08313) and the State of California ADCC. We wish to thank Brock Riggins and James Gatherwright for technical support, and the Department of Veteran's Affairs.

\section{Authors' affiliations}

J M Olichney, C R Hofstetter, K Foster, L J Thal, R Katzman, Alzheimer's Disease Research Center, University of California, San Diego,

California, USA

C Murphy, Department of Psychology, San Diego State University

L A Hansen, Department of Pathology, University of California, San Diego

Competing interests: none declared

\section{REFERENCES}

1 Doty RL, Riklan M, Deems DA, et al. The olfactory and cognitive deficits of Parkinson's disease: evidence for independence. Ann Neurol 1989;25:166-71.

2 Doty RL. Odor perception in neurodegenerative diseases and schizophrenia. In: Doty RL, eds. Handbook of olfaction and gustation, 2nd ed. New York: Marcel Dekker, 2003:479-502.

3 Murphy C, Morgan CD. Olfactory function and event-related potentials in Alzheimer's disease. In: Iqbal K, Sisodia SS, Winblad B, eds. Alzheimer's disease:advances in etiology, pathogenesis and therapeutics.John Wiley and Sons, 2001:237-51.

4 McShane RH, Nagy Z, Esiri MM, et al. Anosmia in dementia is associated with Lewy bodies rather than Alzheimer's pathology. I Neurol Neurosurg Psychiatry 2001;70:739-43.

5 Ballard C, Jacoby R, Teodoro DS, et al. Neuropathologic substrates of clinical phenotype in dementia with Lewy bodies [abstract]. Neurology 2002;58:A318.

6 Merdes AR, Hansen LA, Jeste DV, et al. Influence of Alzheimer pathology on clinical diagnostic accuracy in dementia with Lewy Bodies. Neurology 2003;60:1586-90.

7 McKeith IG, Galasko D, Kosaka K, et al. Consensus guidelines for the clinical and pathologic diagnosis of dementia with Lewy bodies (DLB): report of the consortium on DLB international workshop. Neurology 1996;47:1113-24.

8 McKeith IG, Ballard CG, Perry RH, et al. Prospective validation of consensus criteria for the diagnosis of dementia with Lewy bodies. Neurology 2000;54:1050-8.

9 Verghese J, Crystal HA, Dickson DW, et al. Validity of clinical criteria for the diagnosis of dementia with Lewy bodies. Neurology 1999;53:1974-82.

10 Lopez OL, Hamilton RL, Becker JT, et al. Severity of cognitive impairment and the clinical diagnosis of AD with Lewy bodies. Neurology 2000;54:1780-7.

11 Gearing M, Mirra SS, Hedreen JC, et al. The Consortium to Establish a Registry for Alzheimer's Disease (CERAD). Part X. Neuropathology confirmation of the clinical diagnosis of Alzheimer's disease. Neurology 1995;45:461-6.

12 American Psychiatric Association. Diagnostic and statistical manual of mental disorders, 3rd ed, revised. Washington DC: American Psychiatric Association, 1987.

13 McKhann G, Drachman D, Folstein M, et al. Clinical diagnosis of Alzheimer's disease: report of the NINCDS-ADRDA Work Group under the auspices of Department of Health and Human Services Task Force on Alzheimer's disease. Neurology 1984;34:939-44.

14 Mirra SS, Heyman A, McKeel D, et al. The Consortium to Establish a Registry for Alzheimer's Disease (CERAD). Part II. Standardization of the neuropathologic assessment of Alzheimer's disease. Neurology 1991:41:479-86.

15 Fahn S, Elton RL. Unified Parkinson's disease rating scale. In: Fahn S Marsden CD, Golstein M, Calne DB, eds. Recent developments in Parkinson's disease, vol 2. Florham Park, NJ: MacMillan Healthcare Information, 1987:153-63.
16 Salmon DP, Butters NM. Neuropsychological assessment of dementia in the elderly. In: Katzman R, Rowe J, eds. Principles of geriatric neurology. Philadelphia: FA Davis, 1992:144-63.

17 Mattis S. Mental status examination for organic mental syndrome in the elderly patient. In: Bellack L, Karasu TB, eds. Geriatric psychiatry. New York: Grune and Stratton, 1976:77-120.

18 Robins LN, Helzer JE, Croughan J, et al. National Institute of Mental Health Diagnostic Interview Schedule. Its history, characteristics and validity. Arch Gen Psychiatry 1981;38:381-9.

19 Frye RE, Schwartz BS, Doty RL. Dose-related effects of cigarette smoking on olfactory function. JAMA 1990;263:1233-6.

20 Galasko D, Hansen LA, Katzman R, et al. Clinical-neuropathological correlations in Alzheimer's disease and related dementias. Arch Neurol 1994;51:888-95.

21 Cain WS, Gent JS, Catalanotto FA, et al. Clinical evaluation of olfaction. Am J Otolaryngol 1983;4:252-6.

22 Murphy C, Gilmore MM, Seery CS, et al. Olfactory thresholds are associated with degree of dementia in Alzheimer's disease. Neurobiol Aging 1990;11:465-9.

23 Hansen LA, Samuel W. Criteria for Alzheimer's disease and the nosology of dementia with Lewy bodies. Neurology 1997:48:126-32.

24 Hansen L, Galasko D, Samuel W, et al. Apolipoprotein-E epsilon-4 is associated with neurofibrillary pathology in the Lewy body variant of Alzheimer's disease. Neurosci Lett 1994;182:63-5

25 Braak H, Braak E. Neuropathological staging of Alzheimer-related changes. Acta Neuropathol (Berl) 1991;82:239-59.

26 Stern Y, Alexander GE, Prohovnik I, et al. Inverse relationship between education and parietotemporal perfusion deficit in Alzheimer's disease. Ann Neurol 1992;32:371-5

27 Doty RL, Deems DA, Stellar S. Olfactory function in parkinsonism: a general deficit unrelated to neurologic signs, disease stage, or disease duration. Neurology 1988;38:1237-44.

28 Hendriks AP. Olfactory dysfunction. Rhinology 1988;26:229-51.

29 Murphy C, Schubert C, Cruickshanks K, et al. Prevalence of olfactory impairment in older adults. JAMA 2002;18:2307-12.

30 Hansen L, Salmon D, Galasko D, et al. The Lewy body variant of Alzheimer's disease: a clinical and pathologic entity. Neurology 1990;40:1-8.

31 Braak H, Del Tredici K, Rub U, et al. Staging of brain pathology related to sporadic Parkinson's disease. Neurobiol Aging 2003;24:197-21 1.

32 Hansen LA, Masliah E, Galasko D, et al. Plaque-only Alzheimer disease is usually the Lewy body variant, and vice versa. J Neuropathol Exp Neurol 1993;52:648-54

33 Nordin S, Almkvist O, Berglund B, et al. Olfactory dysfunction for pyridine and dementia progression in Alzheimer's disease. Arch Neurol 1997;54:993-8.

34 Petersen RC, Smith GE, Waring SC, et al. Mild cognitive impairment: clinical characterization and outcome. Arch Neurol 1999;56:303-8.

35 Morgan CD, Nordin S, Murphy C. Odor identification as an early marker for Alzheimer's disease: impact of lexical functioning and detection sensitivity. J Clin Exp Psychol 1995; 17:793-803.

36 Serby M. Olfaction and Alzheimer's disease. Biol Psychiatry 1986;10:579-86.

37 Murphy C, Bacon AW, Bondi MW, et al. Apolipoprotein E status is associated with odor identification deficits in nondemented older persons. Ann NY Acad Sci 1998;855:744-50.

38 Calhoun-Haney R, Murphy C. Apolipoprotein $\epsilon 4$ is associated with more rapid decline in odor identification than in odor threshold or dementia rating scale scores. Brain Cogn (in press)

39 Devanand MD, Michaels-Marston KS, Liu XL, et al. Olfactory deficits in patients with mild cognitive impairment predict Alzheimer's disease at followup. Am J Psychiatry 200, 157:1399-405.

40 Bailie J, Gilbert P, Barr PJ, et al. Odor identification deficits in Lewy Body disease. J Int Neuropsychol Soc 2003;9:164-5.

41 Westervelt $\mathbf{H}$, Stern R, Tremont G. Odor identification deficits in Diffuse Lewy Body Disease. Cogn Behav Neurol 2003;16:93-9.

42 Murphy C, Anderson JA, Markison S. Psychophysical assessment of chemosensory disorders in clinical populations. In: Kurihara K, Suzuki N, Ogawa H, eds. Olfaction and taste XI. Berlin: Springer Verlag 1994:609-13. 